\title{
Perceptual Image Segmentation Using Fuzzy-Based Hierarchical Algorithm and Its Application to Dermoscopy Images
}

\author{
J. Maeda, A. Kawano, S. Yamauchi, Y. Suzuki \\ Department of Computer Science and Systems Eng. \\ Muroran Institute of Technology \\ Muroran 050-8585, Japan \\ Email: junji@csse.muroran-it.ac.jp
}

\author{
A. R. S. Marçal, T. Mendonça \\ Departement of Applied Mathematics \\ University of Porto \\ Rua do Campo Alegre, 687 4169-007 Porto, Portugal \\ Email: andre.marcal@fc.up.pt
}

\begin{abstract}
This paper proposes perceptual segmentation of natural color images using a fuzzy-based hierarchical algorithm and its application to the segmentation of dermoscopy images. A fuzzy-based homogeneity measure makes a fusion of the color features and the texture features. The proposed hierarchical segmentation method is performed in four stages: simple splitting, local merging, global merging and boundary refinement. The effectiveness of the proposed method is confirmed through computer simulations that demonstrate the applicability of the proposed method to the segmentation of natural color images and dermoscopy images.
\end{abstract}

\section{INTRODUCTION}

Image segmentation is a process to partition an image into meaningful regions and is an important step before an image recognition process. In this paper, we are concerned with perceptual image segmentation. Perceptual segmentation is defined to obtain segmentation that produces a small number of segmented regions, and each region should represent a main object or a meaningful part of an object without paying much attention to region interiors. For example, perceptual segmentation could regard a tree that has many branches and leaves as one object in contrast to conventional detailed segmentation. For many applications such as image recognition or image retrieval, it is preferable to obtain such perceptual segmentation with a small number of segmented regions.

Though there is an extensive literature on image segmentation, the papers on perceptual segmentation are limited. Among them, Mirmehdi and Petrou [1] proposed the method based on the multiscale perceptual tower and the probabilistic relaxation method. Shi and Malik [2] proposed the perceptual grouping method based on graph theory, and $\mathrm{Ma}$ and Manjunath [3] proposed the technique based on the Gabor filters and the EdgeFlow. Carson et al. [4] proposed the Blobworld representation based on expectation-maximization algorithm, and Chen et al. [5] proposed the approach based on the adaptive clustering algorithm and the steerable filter decomposition. Although these methods perform well, their algorithms are complicated and it is not so easy to get an optimal segmentation result.
We have proposed a novel technique, a fuzzy-based hierarchical algorithm for perceptual segmentation of natural color images, that is capable of deciding the optimal segmentation result driven by the number of segmented regions [6], [7]. Since the proposed algorithm has the significant advantage to produce the segmentation results by reducing the number of segmented regions one by one at each step, the user is able to determine the optimal result easily just by observing the several-segmented results. In other words, the proposed algorithm has the ability to stop the process at the specified number of segmented regions.

In this paper we investigate the applicability of the proposed segmentation algorithm to the segmentation of dermoscopy images. Dermoscopy is a non-invasive diagnostic technique for the in vivo observation of pigmented skin lesions used for dermatology. This diagnostic tool allows for a better visualization of surface and subsurface structures and permits the recognition of morphologic structures not visible by the naked eye, thus opening a new dimension of the clinical morphologic features of pigmented skin lesions [8]. The purpose here is to identify (segment) the lesion and separate it from the background. Though a number of segmentation methods have been proposed for this purpose [9]-[12], the correct segmentation is still a difficult problem due to the great variety of dermoscopy images. The proposed segmentation algorithm is fitted for the segmentation of dermoscopy images because the final number of segmented regions is expected to be two in most of dermoscopy images.

Several experiments are made to confirm the effectiveness of the proposed method. At first, we apply the proposed method to perceptual segmentation of natural color images contained in the Berkley dataset [13], and compared our results with human segmentation. Secondly, we apply the proposed method to the segmentation of dermoscopy images to investigate the applicability of the proposed algorithm.

\section{Overview of the Segmentation Procedure}

The proposed fuzzy-based hierarchical segmentation procedure is shown in Fig. 1. The proposed algorithm is based 


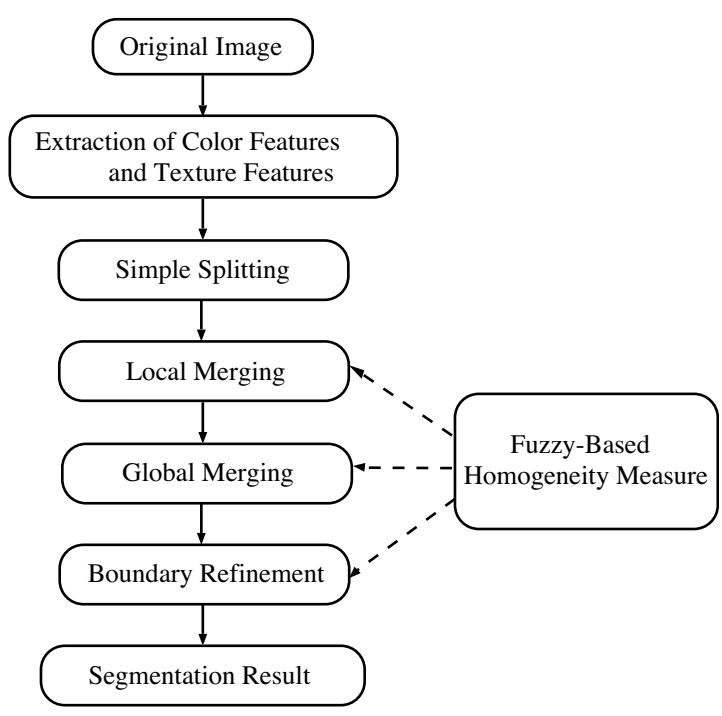

Fig. 1. Block diagram of the segmentation procedure.

on the method of Ojala and Pietikäinen [14]. We drastically improved their algorithm in the following five points for the capability of perceptual segmentation of color images.

1) We adopt the Statistical Geometrical Features (SGF) [15] as texture measures, because the SGF enable the algorithm to set a small minimum block size of $4 \times 4$ and the SGF can remarkably discriminate various types of textures.

2) We adopt fuzzy reasoning [16] to incorporate color features as well as texture features that enables the algorithm to treat color images.

3) We change the stage of hierarchical splitting into simple splitting to reduce the computational cost and the number of parameters.

4) We introduce a new stage of local merging that merges adjacent regions locally in order to significantly reduce the number of regions to be used at the stage of global merging. 5) We redefine the merger importance value at a stage of global merging to stop this stage at the specified number of segmented regions.

As a result of these improvements, the proposed algorithm has the capability of perceptual segmentation using a simple algorithm with an easy implementation that has four hierarchical stages: simple splitting, local merging, global merging and boundary refinement. During the latter three stages, we measure the similarity of any adjacent regions using the fuzzy-based homogeneity measure that combines the similarity of color features and texture features with different degrees of importance. We use the $L^{\star} a^{\star} b^{\star}$ color space to represent color features and the SGF as texture features. The adoption of a fuzzy-based homogeneity measure simplifies the complex mechanism of integrating different features by means of symbolic representations. It also reduces the difficulty in choosing the parameters inherent in segmentation methods, though the tuning of the fuzzy membership functions is still required for each image in the proposed algorithm.

\section{COlOR AND TeXture FEATURES}

\section{A. $L^{\star} a^{\star} b^{\star}$ Color Features}

The $L^{\star} a^{\star} b^{\star}$ color space is a perceptually uniform color space, in which $L^{\star}$ represents brightness and $a^{\star}$ and $b^{\star}$ represent chromatic information. We obtain the $L^{\star} a^{\star} b^{\star}$ color space from the $R G B$ color space, and then the three components, $L^{\star}, a^{\star}$ and $b^{\star}$, are normalized and used as three color features.

\section{B. SGF Texture Features}

The SGF [15] are a set of texture features based on the statistics of geometrical properties of connected regions in a sequence of binary images obtained from an original image. The extraction of the SGF starts by thresholding each component $C$ (where $C=L^{\star}, a^{\star}$ and $b^{\star}$ ) of a color image with a threshold value $\alpha$ that produces numbers of binary images $C_{b}$ defined as

$$
C_{b}(x, y ; \alpha)=\left\{\begin{array}{cc}
1 & C(x, y) \geq \alpha \\
0 & \text { otherwise }
\end{array}\right.
$$

where $1 \leq \alpha \leq n_{l}-1$ and $n_{l}$ is the maximum gray level of each component.

Each binary image $C_{b}(x, y ; \alpha)$ comprises several connected regions. The number of connected regions of 1 -valued pixels and that of 0 -valued pixels give two geometrical measures, $N O C_{1}(\alpha)$ and $N O C_{0}(\alpha)$, respectively. Each connected region is also described by its irregularity (or non-circularity) related to the maximum radius of pixels within the connected region $I$. The irregularity of the $i$ th connected region of 1 valued pixels is defined as

$I R G L_{1}(i, \alpha)=\frac{1+\sqrt{\pi} \max _{i \in I} \sqrt{\left(x_{i}-\bar{x}\right)^{2}+\left(y_{i}-\bar{y}\right)^{2}}}{\sqrt{|I|}}-1$,

where $\bar{x}, \bar{y}$ are the center of mass and $|I|$ is the region's area. Variations in the value of $\operatorname{IRG} L_{1}(i, \alpha)$ from 0 to 1 represent the change in shape of the connected region from circle to line. Then, the average (weighted by size) of the irregularity of 1 -valued pixels in the binary image $C_{b}(x, y ; \alpha)$ gives a irregularity measure

$$
\overline{I R G L}_{1}(\alpha)=\frac{\sum_{i \in I}\left(N O P_{1}(i, \alpha) \cdot I R G L_{1}(i, \alpha)\right)}{\sum_{i \in I}\left(N O P_{1}(i, \alpha)\right)},
$$

where $N O P_{1}(i, \alpha)$ is the number of pixels in the $i$ th connected region of 1 -valued pixels. $\overline{I R G L_{0}}(\alpha)$ is similarly defined.

Each of these four functions, i.e., $N_{1} C_{1}(\alpha), N O C_{0}(\alpha)$ ), $\overline{I R G L_{1}}(\alpha)$ and $\overline{I R G L_{0}}(\alpha)$, is further characterized using the three statistics over an entire image: the average value, the sample mean and the sample standard deviation. This gives 12 SGF for each component of a color image, and thus a total of $36 \mathrm{SGF}$ for one image. At the implementation, a sliding overlapping window of size $5 \times 5$ is used to calculate the SGF of each pixel of an original image. 


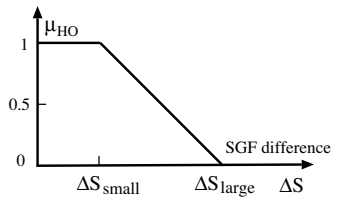

(a)

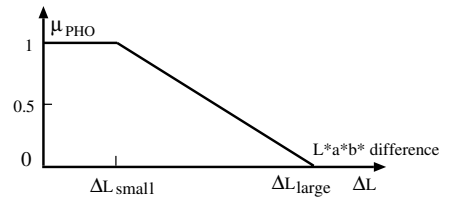

(b)
Fig. 2. Fuzzy membership functions of (a) SGF difference $(\Delta S)$ and (b) $L^{\star} a^{\star} b^{\star}$ difference $(\Delta L)$.

\section{FuZZy-BAsed Homogeneity Measure}

Homogeneity is a measure to test the similarity of two regions under consideration during the segmentation procedure. We adopt a fuzzy-based homogeneity measure to integrate the different features: the $L^{\star} a^{\star} b^{\star}$ color features and the SGF texture features. We use the following fuzzy rules in which each rule has a corresponding membership function.

1) Rule 1: If SGF difference is SMALL, Then HOMOGENEOUSE (HO); Else NOT_HOMOGENEOUSE (NHO).

2) Rule 2: If $L^{\star} a^{\star} b^{\star}$ difference is SMALL, Then PROBABLY_HOMOGENEOUSE (PHO); Else PROBABLY_ NOT_ HOMOGENEOUSE (PNHO).

These fuzzy rules give the SGF texture features a higher priority than the $L^{\star} a^{\star} b^{\star}$ color features because we consider that the texture features provide more important information in perceptual segmentation of textured color images. In these fuzzy rules, a SGF difference is the Euclidean distance of 36 SGF between two regions under consideration, and a $L^{\star} a^{\star} b^{\star}$ difference is the Euclidean distance of three-color components between them.

Four conditions $\mathrm{HO}, \mathrm{NHO}, \mathrm{PHO}$ and $\mathrm{PNHO}$ represent different grades of homogeneity between two regions. Their homogeneity values $\mu_{H O}, \mu_{N H O}\left(=1-\mu_{H O}\right), \mu_{P H O}$ and $\mu_{P N H O}\left(=1-\mu_{P H O}\right)$ can be obtained from fuzzy membership functions of the SGF difference and the $L^{\star} a^{\star} b^{\star}$ difference as shown in Fig. 2. Here $\Delta S$ represents the SGF difference and $\Delta L$ represents the $L^{\star} a^{\star} b^{\star}$ difference. The values of $\Delta S_{\text {small }}$, $\Delta S_{\text {large }}, \Delta L_{\text {small }}$ and $\Delta L_{\text {large }}$ in Fig. 2 have to be tuned empirically.

After fuzzification by applying the above two rules, minmax inference takes place using the fuzzy sets shown in Fig. 3. Then the conventional centroid defuzzification method is applied. Suppose the homogeneity limit is set to be 0.5 . Then, if the inferred homogeneity measure is over 0.5 , the two regions being concerned are regarded as homogeneous and they are merged. We use the value of the homogeneity measure $H$ in the proposed segmentation algorithm.

\section{Segmentation Algorithm}

In the following, we will demonstrate the progress of the proposed segmentation algorithm for a $300 \times 300$ natural color image shown in Fig. 4(a).

\section{A. Simple Splitting}

Ojala and Pietikäinen [14] used the hierarchical splitting algorithm that recursively splits an original image into square

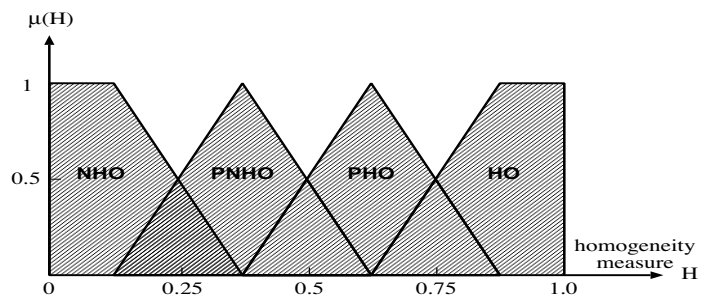

Fig. 3. The fuzzy sets used for homogeneity inference.

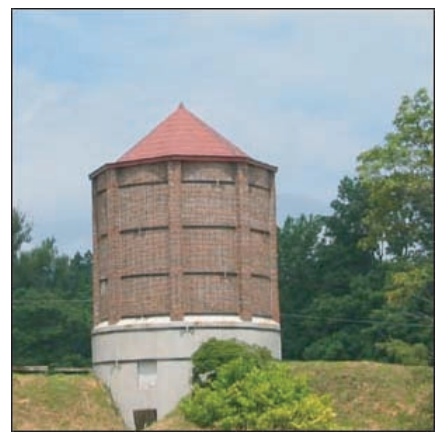

(a)

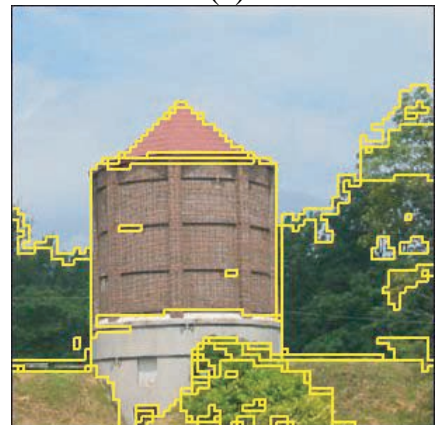

(c)

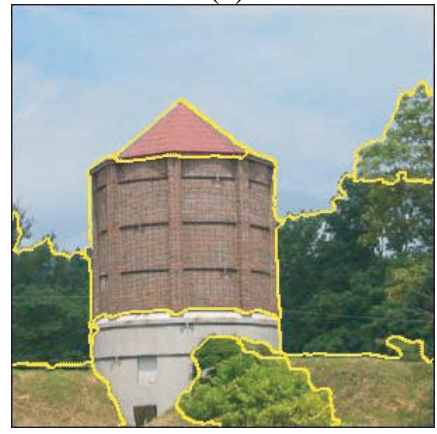

(e)

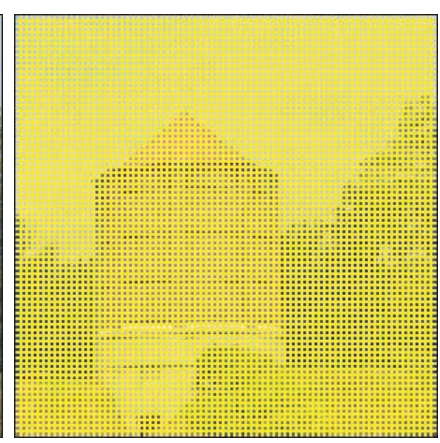

(b)

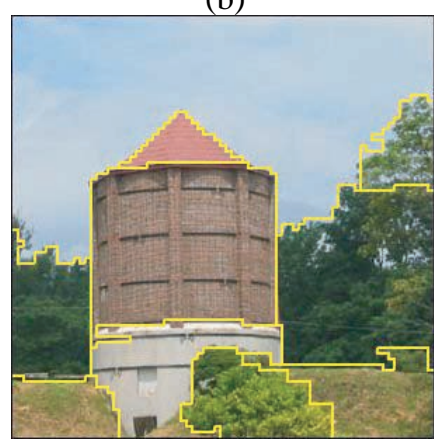

(d)

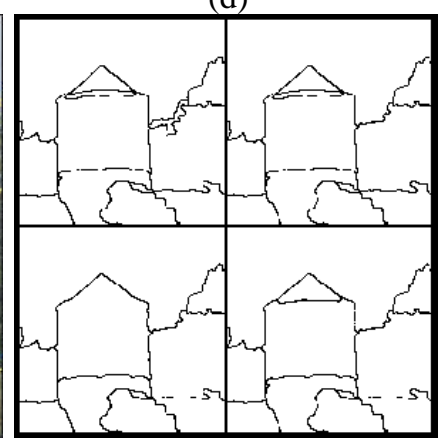

(f)
Fig. 4. Progress of the proposed segmentation algorithm: (a) original color image; (b) result of simple splitting; (c) result of local merging; (d) result of global merging with $N=10$; (e) result of boundary refinement; (f) results of segmentation with $N=12,11,10$ and 9 shown clockwise from the upper left.

blocks of varying sizes. Since their algorithm requires computational cost and the threshold value, we changed the stage of hierarchical splitting into simple splitting to reduce the computational cost and the number of parameters. In simple splitting, an original image is divided into rectangular subblocks of size $4 \times 4$ as shown in Fig. 4(b). It is noted the adoption of the 
SGF texture features and the incorporation of local merging in our algorithm enable the use of simple splitting.

\section{B. Local Merging}

Local merging is a newly proposed stage by us to merge adjacent regions locally for drastically reducing the number of regions to be used at the stage of global merging. The SGF of each $4 \times 4$ subblock are obtained by averaging the texture features of all pixels within the subblock, so does the $L^{\star} a^{\star} b^{\star}$ color features of each subblock.

The homogeneity between any current region and its neighboring adjacent region is measured individually. Then the two adjacent regions having the largest homogeneity measure $H_{\max }$ are regarded as similar and they are merged to become one region if the value of $H_{\max }$ is higher than a threshold 0.5. The process is continued until all regions are scanned. We set the threshold 0.5 to avoid over merging in this stage. The result of local merging is shown in Fig. 4(c).

\section{Global Merging}

Global merging is a stage to merge similar adjacent regions globally. A pair of adjacent regions with the smallest merger importance value among all possible mergers in an entire image will be merged at each step. We define merger importance $(M I)$ as the ratio of the number of pixels in the smaller region to the homogeneity measure of adjacent regions

$$
M I=\frac{P_{\text {small }}}{H} .
$$

This procedure finds the best possible pair of adjacent regions globally whose merging introduces the smallest change in the segmented image. Since global merging reduces the number of segmented regions one by one at each step and merger importance removes the less important regions first, the essential regions remain to the end and thus perceptual segmentation is achieved.

It is also easy to stop the algorithm at the specified number of segmented regions $N$. Fig. 4(d) shows the result of global merging when we set $N=10$ to obtain perceptual segmentation.

\section{Boundary Refinement}

Boundary refinement is finally performed to smooth the jagged boundaries after global merging. If an image pixel is on the boundary of at least two distinct regions, a discrete disk with radius 3 will be placed on it. Then the homogeneity measure $H$ between the disk and its neighboring region is calculated individually to decide if the pixel needs to be relabeled. The next scan will check the neighborhoods of the relabeled pixels until no pixels are relabeled. The result of boundary refinement is shown in Fig. 4(e).

In the practical implementation of the proposed algorithm, we can easily choose the desirable optimal result with an appropriate roughness by observing the several-segmented results. The results of segmentation when we set $N=12$, 11, 10 and 9 are shown clockwise from the upper left in Fig. 4(f). This figure demonstrates how the number of segmented regions decreases in the proposed algorithm.

\section{EXPERIMENTAL RESULTS}

In this section, we present experimental results to assess the performance of the proposed segmentation method. Since we set $\Delta S_{\text {small }}=0$ and $\Delta L_{\text {small }}=0$ in the proposed algorithm, we have to tune two parameters $\Delta S_{\text {large }}$ and $\Delta L_{\text {large }}$ of the fuzzy membership functions in Fig. 2 differently according to each image and they were determined empirically.

At first, we apply the proposed method to several $481 \times 321$ natural color images contained in the Berkley dataset [13], and compared our results with human segmentation. Images in Fig. 5(a) show original images and images in Fig. 5(b) represent hand-labeled segmentations (darker contours were marked by more human subjects) both from the Berkley database. The final optimal segmented results of the proposed method are shown in Fig. 5(c). In Fig. 5(c), we only depict the boundaries of the segmented regions for the clarity of comparison with human segmentation.

Comparisons between Fig. 5(b) and (c) clearly indicate that the proposed method has an excellent ability to produce perceptual segmentation that is almost identical to the human segmentations. The proposed method successfully detects the essential objects without paying much attention to region interiors and thus it yields a small number of segmented regions. We have tried to segment numbers of images from the Berkley dataset and it was revealed that the proposed method is good at relatively rougher segmentation than the other perceptual segmentation algorithms. We have also confirmed the advantage of an easy selection of the optimal result that is best suited to user's intention from among the severalsegmented results. On the other hand, the shortcoming of the proposed method is that it is difficult to maintain important but small objects with visual attention, such as eyes of creatures or small halls at the top row and fifth row in Fig. 5(a) and (b), because such small objects tend to disappear at the stage of global merging.

Secondly, we apply the proposed method to the segmentation of dermoscopy images to investigate the applicability of the proposed algorithm. The proposed algorithm was applied to 50 dermoscopy images randomly selected from the clinical database of the Hospital Pedro Hispano, Matosinhos, Portugal [12]. Fig. 6(a)-(d) show 4 out of the 50 original dermoscopy images. These images indicate that the segmentation is not a straightforward task because there is a large variety of lesions, skin types, presence of hair and so forth in dermoscopy images. Since the dark shadows of four corners in the original dermoscopy images give a bad influence upon our algorithm, we have to reject this influence at the stage of local merging.

Ideally, the segmentation of dermoscopy images should be stopped automatically without human decision. This means that the parameters of the algorithm are kept fixed or automatically tuned. Since the proposed algorithm is universal and not optimized for dermoscopy images, we have applied the proposed method with the use of fixed parameters and $N=2$. 

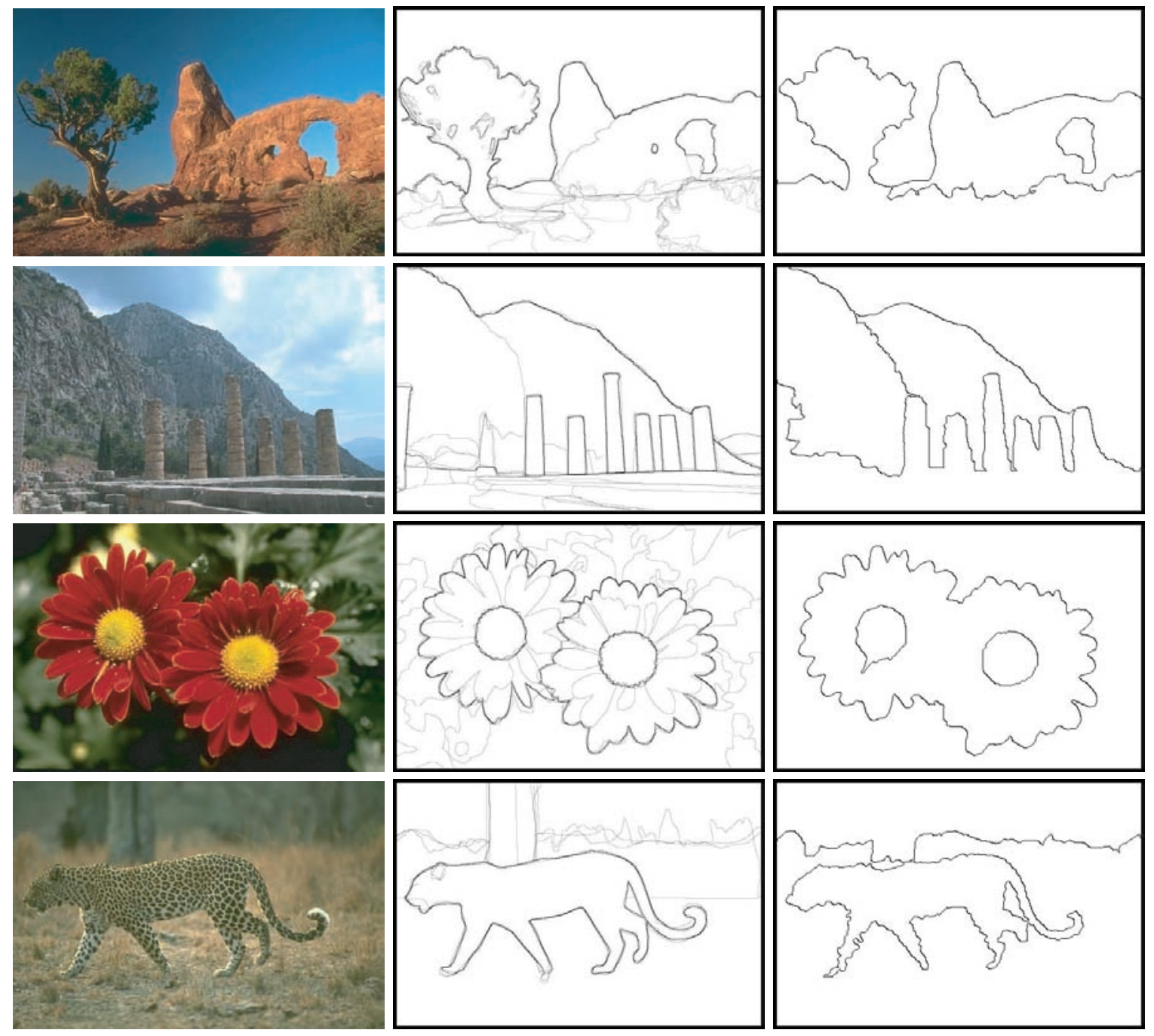

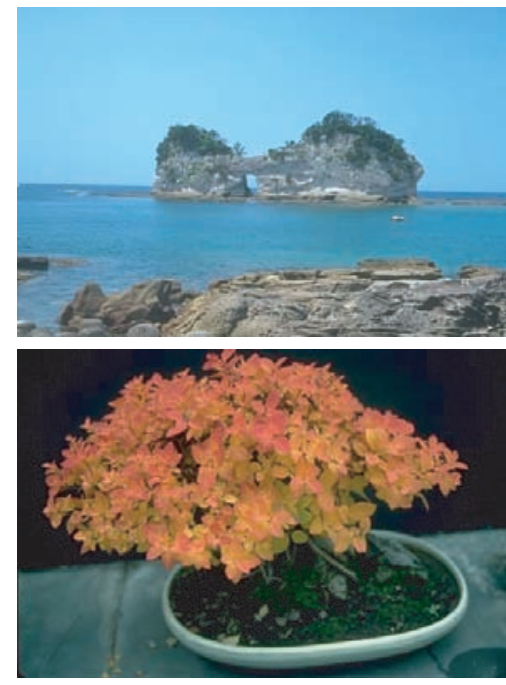

(a)

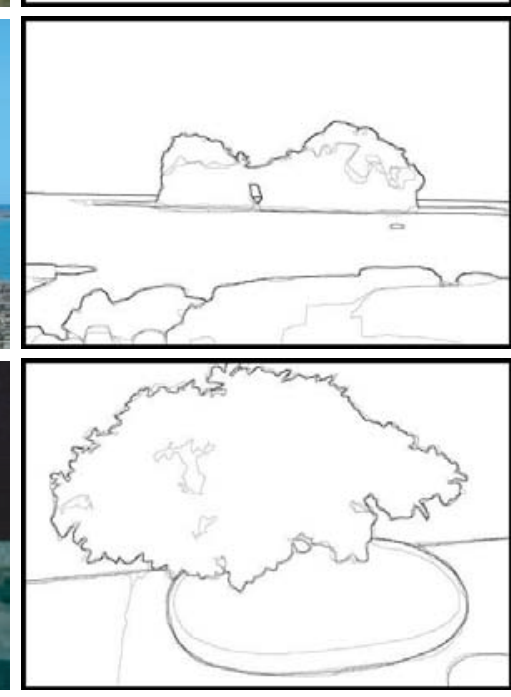

(b)

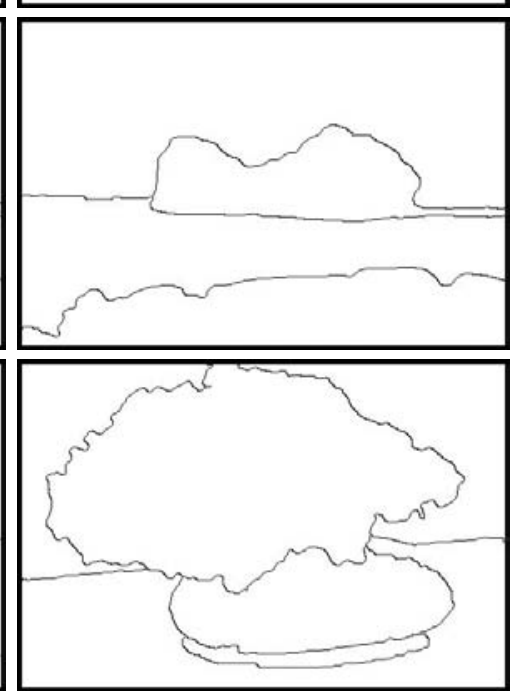

(c)

Fig. 5. Segmentation of natural color images contained in the Berkley dataset: (a) original color image; (b) human segmentation; (c) result of the proposed method. 


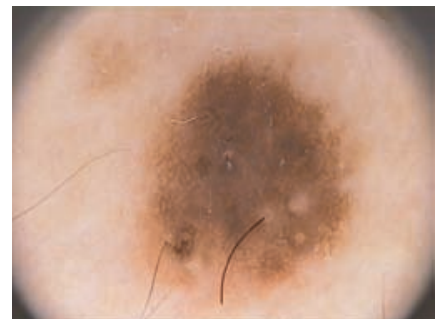

(a)

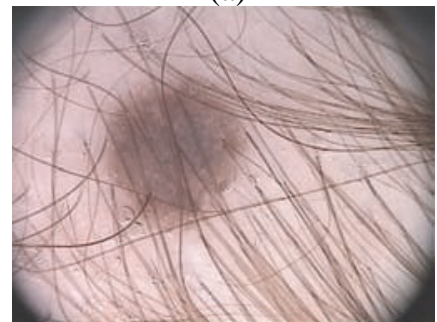

(c)

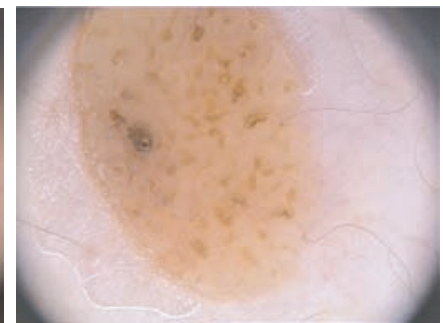

(b)

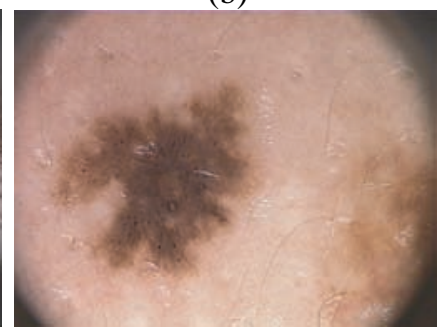

(d)
Fig. 6. The original dermoscopy images.

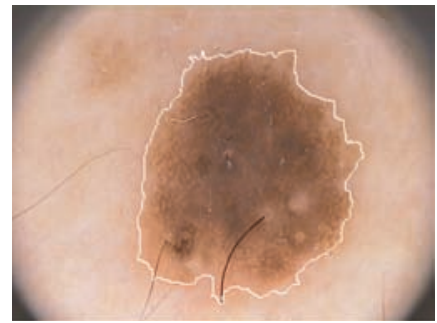

(a)

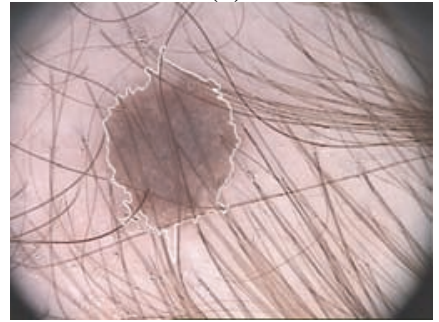

(c)

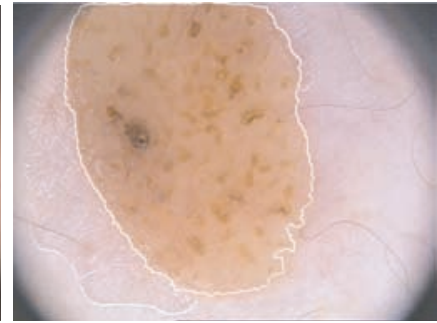

(b)

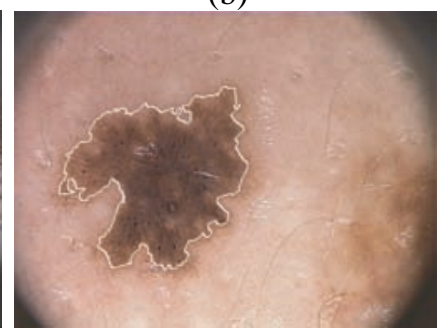

(d)
Fig. 7. Segmentation of dermoscopy images in Fig. 6 using the proposed segmentation algorithm:

We have successfully segmented 45 images out of 50 using the proposed algorithm with automatically tuned parameters. Fig. 7(a)-(d) show the segmentation results of the dermoscopy images in Fig. 6. In Fig. 7, the boundaries are superimposed on the original image to check the accuracy of the proposed algorithm. These results demonstrate the effectiveness and the applicability of the proposed method to segment dermoscopy images. However, further investigations are necessary to precisely compare the proposed algorithm with other methods, and in the future we plan to have a more careful evaluation of the experimental results by a dermatologist.

\section{CONCLUSIONS}

In this paper, we presented a perceptual segmentation of color images using the proposed fuzzy-based hierarchical algorithm. Since the fuzzy-based homogeneity measure makes a reliable fusion of the $L^{\star} a^{\star} b^{\star}$ color features and the SGF texture features, the proposed method provides perceptual segmentation that maintains uniform texture regions and accurate boundaries. The proposed algorithm was applied to the segmentation of natural color images and dermoscopy images and the experimental results demonstrate the effectiveness of the proposed method. Future work should include the automatic tuning of the parameters based on information extracted from original images, and a detailed clinical evaluation by a dermatologist.

\section{ACKNOWLEDGMENT}

The author would like to thank Dr. Jorge Rozeira and Hospital Pedro Hispano for the access to the dermoscopy images.

\section{REFERENCES}

[1] M. Mirmehdi and M. Petrou, "Segmentation of color textures," IEEE Trans. PAMI, Vol. 22, No. 2, pp. 142-159, 2000.

[2] J. Shi and J. Malik, "Normalized cuts and image segmentation," IEEE Trans. PAMI, Vol. 22, No. 8, pp. 888-905, 2000.

[3] W. Y. Ma and B. S. Manjunath, "EdgeFlow: a technique for boundary detection and image segmentation," IEEE Trans. IP, Vol. 9, No. 8, pp. 1375-1388, 2000.

[4] C. Carson, S. Belongie, H. Greenspan, and J. Malik, "Blobworld: Image segmentation using expectation-maximization and its application to image querying," IEEE Trans. PAMI, Vol. 24, No. 8, 1026-1038, 2002.

[5] J. Chen, T. N. Pappas, A. Mojsilovic, and B. E. Rogowitz, "Adaptive perceptual color-texture image segmentation," IEEE Trans. IP, Vol. 14, No. 10, pp. 1524-1536, 2005.

[6] J. Maeda, K. Kawano, S. Saga, and Y. Suzuki, "Unsupervised perceptual segmentation of natural color images using fuzzy-based hierarchical algorithm," in Springer Lecture Notes in Computer Science, Vol. 4522, 2007, pp. 462-471.

[7] J. Maeda, K. Kawano, S. Saga, and Y. Suzuki, "Number-driven perceptual segmentation of natural color images for easy decision of optimal result," in Proc. IEEE Int. Conf. on Image Processing, 2007, Vol. 2, pp. II-265-II-268.

[8] G. Argenziano, H. P. Soyer, V. DeGiorgi, et al., Dermoscopy an interactive atlas, Milan, Italy: EDRA Medical Publishing, 2000.

[9] P. Schmid, "Segmentation of digitalized dermatoscopic images by twodimensional color clustering," IEEE Trans. Med. Imaging, Vol. 18, No. 2, pp. 164-172, 1999.

[10] H. Ganster, A. Pinz, R. Roehrer, E. Wilding, M. Binder, and H. Kitter, "Automated melanoma recognition," IEEE Trans. Med. Imaging, Vol. 20, No. 3, pp. 233-239, 2001.

[11] P. Schmid-Saugeon, J. Guillod, and J. P. Thiran, “Towards a computeraided diagnosis system for pigmented skin lesions," Comput. Med. Imaging Graph., Vol. 27, No. 1, pp. 65-78, 2003.

[12] T. Mendonça, A.R. S. Marçal, A. Vieira, J. C. Nascimento, M. Silveira, J. S. Marques, and J. Rozeira, "Comparison of segmentation methods for automatic diagnosis of dermoscopy images," in Proc. 29th Annual Int. Conf. of IEEE Engineering in Medicine and Biology Society, 2007, pp. 6572-6575.

[13] D. Martin, C. Fowlkes, D. Tal, and J. Malik, "A database of human segmented natural images and its application to evaluating segmentation algorithms and measuring ecological statistics," in Proc. IEEE Int. Conf. on Computer Vision, Vol. 2, pp. 416-423, 2001. Available from: www.eecs.berkeley.edu/Research/Projects/CS/vision/grouping/segbench/

[14] T. Ojala and M. Pietikäinen, "Unsupervised texture segmentation using feature distributions," Pattern Recognition, Vol. 32, No. 3, pp. 477-486, 1999

[15] Y. Q. Chen, M. S. Nixon, and D. W. Thomas, "Statistical geometrical features for texture classification," Pattern Recognition, Vol. 28, No.4, pp. 537-552, 1995.

[16] L. A. Zadeh, "Fuzzy sets," Inform. Control, Vol. 8, pp. 338-353, 1965. 